\title{
Modern European Monographs for Quality Control of Chinese Herbs
}

Authors

Affiliations
Rudolf Bauer ${ }^{1}$, Gerhard Franz ${ }^{2}$

${ }^{1}$ Institute of Pharmaceutical Sciences, TCM Research Center, Graz, Austria

${ }^{2}$ Department of Pharmacy, University of Regensburg, Regensburg, Germany
Key words

- TCM

- traditional Chinese medicine

- herbal medicinal products

- quality control

- pharmacopoeia

- monographs

\section{Abstract \\ $\nabla$}

The actual concern about the safety and efficacy of herbal drugs originating from traditional Chinese medicine (TCM) is based on observations that these medicinal plants may have a high risk potential due to insufficient definitions, problems with identity, purity and falsifications. No uniform legal status for these groups of herbal drugs currently exists in the European Union. For quality control, monographs for TCM herbs can mainly be found in the Pharmacopoeia of the Peoples Republic of China. Based on these facts the Commission of the European Pharmacopoeia de-

\section{Introduction}

$\nabla$

Herbal drugs have a long tradition as medicines in the European market and are recognised within the regulatory framework on medicinal products. Nonetheless their importance and economic impact varies from country to country [1]. Like all kinds of medicines, herbal drugs and the products made thereof have to prove their quality, efficacy and safety prior to gaining access to the market [2].

The quality parameters of herbal drugs are usually specified and implemented in the respective pharmacopoeias, either in national ones or in the European Pharmacopoeia (EP), which is currently published in the 7th edition. The EP arose out of the need for a transnational pharmacopoeia in Europe, in which different states could agree and mutually accept the best common standards for medicinal substances [3]. The European Pharmacopoeia Convention has been signed by 27 states and by the Commission of the European Communities. Moreover, 22 countries and WHO have observer status $[4,5]$. Therefore, the member states are exceeding the 27 countries of the European Union. The EP is elaborated and published by the cided in 2005 to establish TCM-herbal drug monographs for the most important medicinal plants imported from Far East. These new monographs had to be established and evaluated on the basis of existing monographs in the Chinese Pharmacopoeia (ChP), English edition 2005. Due to important differences in the overall features of EP and ChP, a simple adapt/adopt procedure was not feasible. Therefore, specialist groups were mandated with a corresponding working programme. Some results and actual problems related to this working programme will be presented and discussed.

European Directorate for the Quality of Medicines \& Health Care in Strasbourg, which is an institution of the Council of Europe [4]. The monographs in the EP are addressed to the respective national authorities, manufacturers and also to public or community pharmacies.

In 1997, the European Parliament stated in document A4-0075/97 on the issue of incorporating nonconventional medical remedies to the European Pharmacopoeia: "The European Pharmacopoeia, as drawn up by the Council of Europe, needs to be opened up to other pharmacopoeiae particularly the medicinal plants used in Chinese medicine." The activities of the EP to include traditional ethic medicines started in 2005, when the respective commission published a statement as follows: "Monographs on herbal drugs used in traditional Chinese medicine (TCM) should be developed to give a modern quality standard according to European Pharmacopoeia in principles and to facilitate and encourage use by practitioners for safe, authorised products".

In this initial phase, only TCM-herbal drugs were considered for an elaboration process. Others, such as products originating from Ayurvedic medicine or South American medicines were not 
included in the initial working programme, since at the time, other ethic medicines had a much lesser impact in the EU member states.

The inclusion criteria for a defined working programme by the EP Commission were based on the following priority setting:

- Volume of use

- Known problems of quality

- Known problems of toxicity

- Risk of substitution with toxic herbal drugs

- Existence of a monograph on a closely related herbal drug. Thus, the final working programme was established by a procedure, defined by the EP Commission as follows:

- Decision on a list of herbal drugs to be included

- Monographs available from national European pharmacopoeias should be published immediately as drafts in Pharmeuropa

- Priority in the evaluation procedure should be given for precise identification such as macroscopic examination, microscopic examination and TLC fingerprinting

- Assay methods should be included if existing, if not currently available, a method should be developed if necessary and as second priority.

Currently, the EP contains approximately 2100 monographs out of which are 166 herbal drugs and 105 herbal drug preparations [2]. In this context it is important to state that the traditional term "herbal drug" used in the EP is synonymous with the term "herbal substance" used by the European Community regulation (EMA).

In the section "Herbal Drug Preparations", 28 extracts, 15 tinctures and 31 essential oils are included at the moment. In addition, 21 "general methods" in pharmacognosy are implemented and further a series of "general chapters".

Finally, one important decision has been made by the EP Commission in 2009, whereby starting with the 7th edition of the EP, all monographs of "Herbal Drugs" and "Herbal Drug Preparations" will be published in a separate chapter with the reasoning to have an easier identification and better handling of the respective herbal items. In consequence, also monographs on TCM herbal drugs and the respective "General Methods" will be located in this chapter of the EP.

\section{Traditional Chinese Medicines for the European Pharmacopoeia \\ $\nabla$}

The selection of items, i.e., herbal drugs originating from TCM, is not an easy task, since it is known that approximately 5000 herbal, animal and mineral drugs have been used in China [6]. ChP 2005 Vol. I contains 1145 monographs on drugs of herbal, animal and mineral origin. Among them are 538 monographs on herbal drugs. Detailed data for the utilisation of TCM medicines in Europe are not published. It was estimated that in the different EU member states between 75 and 125 herbal drugs of Chinese origin are most frequently used.

Thus, the selection of candidates to be included in the EP Working Programme, i.e., the priority setting, was based - after an inquiry at the respective national authorities - on the following criteria:

- Quality and completeness of existing monographs in the ChP 2005

- Extent of use in the different EU member states

- Risk potential (adulterations, toxicity, poor quality)
- Availability of herbal drug material representative for the current market situation

Out of the more than 100 proposed TCM herbal drugs an initial list of 83 candidates was included into the working programme.

\section{Initial working programme based on the}

proposals of the respective EP member states ( $\bullet$ Table 1 ) The basic concept of the EP Commission intended that the new monographs originating from the respective monographs of the ChP should follow the style and quality parameters of the EP. New monographs should be elaborated according to the current requirements of EDQM for a herbal drug monograph. Following the EDQM "Guide for the Elaboration of Monographs on Herbal Drugs", it should be kept in mind that a monograph on a herbal drug has to be defined with the same overall structure as a monograph on a chemical substance, with the consequence that all tests and assay methods described in a monograph must be validated according to the respective procedures stated in the "Technical Guide" of the EP [7].

One major problem, which had to be taken into account, was the fact that many of the TCM herbal drugs on the market are not used in their genuine form but in a so-called processed state, which, for herbal drugs in Western medicine, is a completely unknown feature. As a consequence, a simple adaption/adoption procedure of existing herbal drug monographs was not feasible.

\section{Apparent Differences between Herbal Drug Monographs in EP6 versus ChP 2005 (O Table 2) $\nabla$}

\section{Definition}

According to the current technical guide of the EP, in the "Definition" section the following information should be provided:

- State of the drug: whole, cut, peeled, fresh or dried

- Complete scientific name of the plant (genus, species, subspecies, variety, author)

- Whenever possible, the minimum content of quantified constituents should be specified

Since nomenclature is not yet harmonised in East Asia, there is an urgent need for a clear definition of the botanical source. For example, "Bianxu" obtained from China mainland is supposed to be Polygonum aviculare (Polygonaceae); obtained from Taiwan it is Euphorbia thymifolia (Euphorbiaceae), and from Hong Kong it may be Belamcanda chinensis (Iridaceae) [8].

A problem to be solved is the inclusion of the original Chinese name of the plant (herbal drug material) citing the Pinyin names in the "Definition" section. The Pinyin names are the originating form, the Hany Pinyin as approved by the People's Republic of China. However, they do not correspond with the Tongyong Pinyin used in Taiwan. When the first TCM herbal drug monographs were ready for publication in the $\mathrm{EP}$, the Chinese titles, taken from the ChP, both, as sinograms and Pinyin, were included in the "Definition". Unfortunately, this was not accepted by the EP Commission with the reasoning that in Chinese different spellings exist for the same herbal drug. However, it was agreed that the Chinese names are kept in the monographs until final publication. But since the Chinese names of the individual herbal drug are an essential piece of information for the user, a solution for this problem has to be found.

In TCM herbal drug monographs the specification of a minimum content of active constituents is not always obvious, because pharmacological data of therapeutically relevant compounds, as 
Table 1 Initial working programme based on the proposals of the respective EP member states.

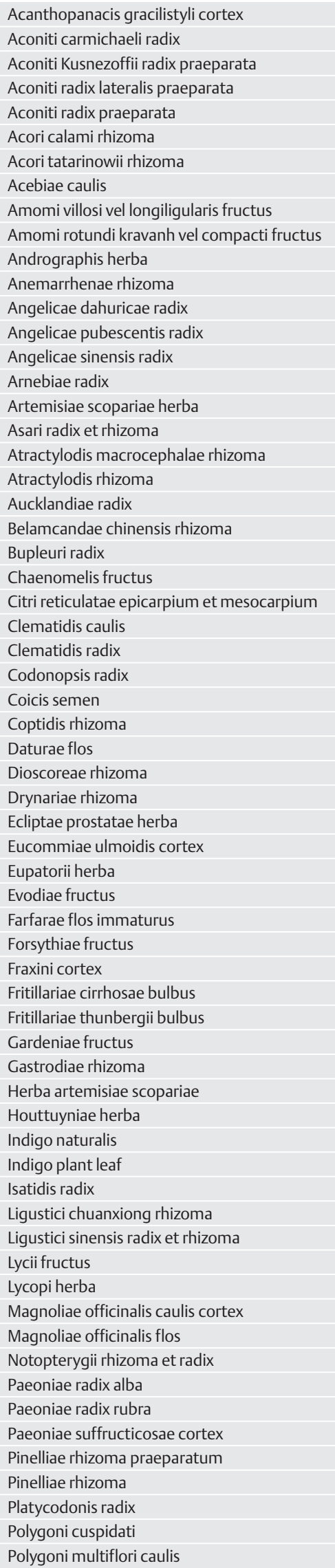

Table 1 Initial working programme based on the proposals of the respective EP member states. (continued)

Polygoni multiflori radix

Polygoni multiflori radix praeparata

Polygoni orientalis fructus

Polygoni tinctorii folium

Poria

Rehmanniae radix

Rhei radix et rhizoma

Salviae Miltiorrhizae radix et rhizoma

Saposhnikoviae radix

Scutellariae baicalensis radix

Sinomenii acuti caulis

Uncariae cum uncis ramulus

Zanthoxyli pericarpium

Ziziphi spinosae semen

Table 2 Apparent differences between herbal drug monographs in EP 2008 versus ChP 2005.

\section{EP 2008}

CP 2005 (Engl. Edition)

1. Nomenclature:

- Engl. title

Latin title

1. Chinese Title

Latin Title

(Translated Engl.Title)

2. Definition:

2. Source

- state of the drug: whole, fragmented

3. Formulary

4. Processing

- scientific botanical name

- part of the plant used

- stage in the growth cycle when harvesting takes place

- minimum content of quantified constituents

- statement: "dried drug"

5escription

6. Identification

7. Inspection

8. Extractives

9. Assay

10. Processing of crude drug

11. Action and indications

3. Characters:

- not mandatory

4. Identification

A) Macroscopic botanical characters

B) Microscopic botanical characters

12. Usage and dosage of powdered drug only

\section{C) TLC [HPTLC]}

no Rf values, but chromatograms described in the form of a table (upper, middle, lower third of the table)

5. Tests:

- classical tests (ash, loss on drying, etc.)

- heavy metals

- pesticides

- aflatoxins

- if necessary: aristolochic acids, pyrrolizidine alkaloids

6. Assay

7. Storage

8. Reagents 
in Western medicine, do not always exist. As for the inclusions of botanical varieties, in many monographs of the ChP a broad spectrum of species and subspecies is listed, which is difficult to treat in a correct way in the "Identification" section. For example, according ChP 2005, Flos Magnoliae can be derived from Magnolia biondii Pamp., M. denudate Desr. and M. sprengeri Pamp. In the case of Zicao, it is still not clear which species is used in practise: Lithospermum erythrorhizon (Zicao), Arnebia euchroma (Ruan Zicao) or Onosma paniculatum (Dian Zicao) [9]. Similar problems exist with "Mutong" (Akebia quinata Decne), "Chuanmutong" (Clematis armandii Franch.) and "Guanmutong" (Aristolochia manshuriensis Kom.) [10]. During elaboration of monographs, it turned out to be very difficult to obtain samples of each variety/ species for the practical evaluation of the tests, assays and limits. An additional problem is related to the "State of the Drug" section, since for many TCM herbal drugs processing is essential. At the moment it is not obvious whether processing methods are going to be included in the "Definition" or whether they will be specified in a separate chapter "Production (Processing)". With respect to the "Character" of the herbal drug, indications about odour, taste and specific colours are not mandatory in the EP, while in the ChP these characteristic data are essential since practical TCM is based upon these indications. However, it must be considered that "taste" may have a different meaning in TCM theory.

\section{Identification}

The "Identification" section in the EP is a very important chapter for all herbal drugs including the new TCM materials. The combination of different methods such as microscopic and macroscopic examination of the botanical characters (Identification $A$ and $B$ ), and TLC fingerprinting (Identification $C$ ) are typical features of EP monographs. Wagner and Bauer [11] have published 75 monographs with TLC and HPLC fingerprints of Chinese herbs so far, which are very useful for the elaboration of the pharmacopoeia monographs.
In community pharmacies, at least Identifications $A$ and $B$ have to be carried out. However, there are striking differences between EP and ChP in the identification part. Often ChP 2005 does not provide microscopic descriptions of the powder, and also no herbal drug powder illustrations, while TLC fingerprinting is also common [12]. However, EP is providing schematic chromatograms of reference substances in comparison to the sequence of compounds in the herbal drug extract ( Fig. 1), while ChP is only describing the separation in words. In both cases, coloured photographs would be desirable. The question whether additional identification methods by molecular biological methods, i.e., CE and DNA fingerprinting [13-16], should be included into the EP, is currently under discussion.

\section{Test}

The "Test" section in the EP contains chapters for the classical test methods, such as "Foreign Matter" (2.8.2.), "Loss on Drying" (2.2.32.), “Water" (2.2.13.), "Pesticides residues" (2.8.13.), "Heavy metals" (2.4.27.), determination of "Total Ash" (2.4.16.), "Ash soluble in hydrochloric acid" (2.8.1.), "Extractable Matter" "Swelling Index” (2.8.4.), “Bitterness Value” (2.8.15.), "Aflatoxin B1" (2.8.18.), "Ochratoxin" (2.8.22.), and "Radioactive Contamination". In addition, microbiological purity has to be tested according the monograph 2.6.31. Microbiological examination of herbal medicinal products for oral use. Recommendations on the microbiological quality of herbal medicinal products consisting solely of one or more herbal drugs are given in the EP chapter 5.1.4. Microbiological quality of pharmaceutical preparations and substances for pharmaceutical use. EP provides tolerance levels for the microbial load of medicinal plants, which are based on intended application and on preparation technique. The accepted microbial load is higher when a plant drug is intended to be treated with boiling water prior to use, as this leads to a decrease of microbial growth. The criteria of EP are not mandatory, but are used as a recommendation for target levels. The limits recommended have been intensively discussed because in some cases it is rather difficult
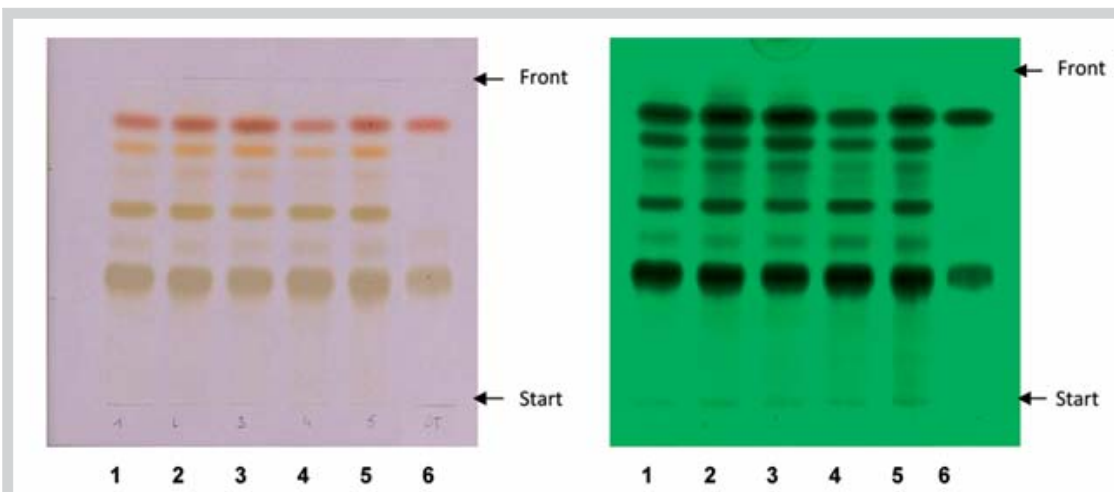

Fig. 1 TLC test and schematic chromatograms of Salviae miltiorrhizae Radix et Rhizoma (Danshen); $1-5=$ different batches of Danshen; 6 = reference solution (salvianolic acid $B$ and tanshinone $\|_{A}$ ).
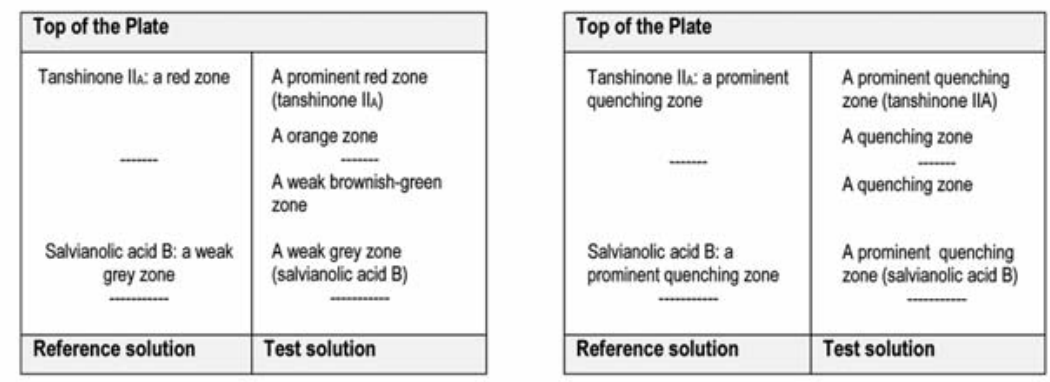


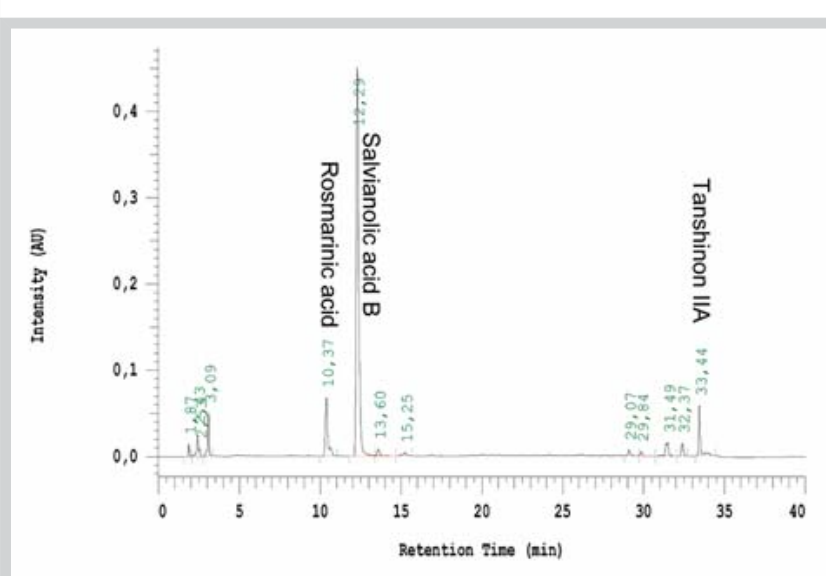

Fig. 2 HPLC separation for the assay of salvianolic acid B and tanshinone $\|_{A}$ in Salviae miltiorrhizae Radix et Rhizoma (Danshen).

to comply with them. Especially organically produced materials may be treated with natural fertilizers such as stinging nettle broth which contains considerably higher microbial levels, and they are also preferred by many insects which often carry contaminants $[17,18]$. Another problem is insufficient drying of the material, which often leads to the growth of moulds. Therefore, according to EP, "Loss on Drying" should be limited to a low percentage [7].

Also the monograph on "Pesticide Residues" (2.8.13.) has recently been updated based on previous experience $[19,20]$. A new monograph on "Heavy metals in herbal drugs and fatty oils" (2.4.27.) has been established. For the EP monograph Herbal drugs the following maximum limits have been set with respect to dry matter: lead $5 \mathrm{mg} / \mathrm{kg}$, cadmium $1.0 \mathrm{mg} / \mathrm{kg}$, mercury $0.1 \mathrm{mg} / \mathrm{kg}$, "unless otherwise stated in an individual monograph or unless otherwise justified and authorised". These tolerance levels have been discussed for a long time [19,21-23].

A test method for aristolochic acids in herbal drugs has recently been developed and implemented in the EP. Aristolochic acids are liver and nephrotoxic, and herbal medicinal products containing these compounds are banned [24]. Nevertheless, there are several Chinese medicinal herbs still on the market, which contain aristolochic acids $[25,26]$. The "Test for Aristolochic Acids in Herbal Drugs" (2.8.21.) contains a Method A as a screening test for aristolochic acids by HPTLC, further Method B as a limit test for aristolochic acid A by HPLC, and finally a Method C as a confirmation test for aristolochic acid A by LC-MS. The urgent need for an elaboration and inclusion of these new test methods was based upon a series of intoxications because of adulterations.

Currently, elaboration and validation of appropriate test methods for the determination of pyrrolizidine alkaloids are in progress and will be implemented in the "Test" programme. Pyrrolizidine alkaloids are highly liver toxic, and therefore limits are urgently needed $[27,28]$. Pyrrolizidine alkaloids occur predominantly in the Compositae family, in plants of the Senecioneae (24 genera, the genus Senecio is prevelant) and Eupatorieae subtribe (mainly in the genera Eupatorium and Ageratum), in virtually all plants of the Boraginaceae family, and in the Fabaceae family (Leguminosae) in the subtribe Crotalariaceae, mainly in the genus Crotalaria, but also in the genera Chromolaena and Lotononis.

In comparison, the "Test" section of the ChP 2005 has been restricted to items such as Foreign Matter, Water, Total Ash, Acid In- soluble Ash, Extractives and a few other methods utilised for some individual monographs. Tests for heavy metals have been occasionally included, like AAS or ICP-MS determination in Panacis quinquefolii Radix (Xi Yang Shen), Paeoniae Radix alba (Bai Shao), Glycyrrhizae Radix (Gan Cao), Salviae miltiorrhizae Radix (Dan Shen), Lonicerae Flos (Jin Yin Hua), and Astragali Radix (Huang Qin). The maximum limits are: lead $5 \mathrm{mg} / \mathrm{kg}$, cadmium $0.3 \mathrm{mg} /$ $\mathrm{kg}$, mercury $0.2 \mathrm{mg} / \mathrm{kg}$, arsenic $2.0 \mathrm{mg} / \mathrm{kg}$, and copper $20.0 \mathrm{mg} /$ $\mathrm{kg}$. Therefore, harmonisation would be desirable.

\section{Assay}

The "Assay" is an important section of all herbal drug monographs, whereby the minimum content of quantified constituents provided in the "Definition" should be determined by appropriate methods. However, for many TCM herbal drugs no constituents with known therapeutic activity (active markers) are known which could be utilised as a standard. In these cases "analytical markers" have to be used and examined with the appropriate methods. In earlier monographs on herbal drugs, assays often have been carried out with global spectrophotometric or titrimetric methods (i.e., determination of flavonoids, or of total alkaloids). For modern monographs, specific determinations of single constituents by HPLC or GLC are widely preferred, although it is known that therapeutic efficacy of herbal drugs is based on several active compounds in most of the cases. Therefore modern concepts of fingerprint analyses are scientifically discussed for Chinese herbs $[29,30]$. However, such methods have not yet been implemented. Instead an assay of a polar and a nonpolar marker may be a surrogate, as it is practised for salvianolic acid B and tanshinone IIA in Salviae miltiorrhizae Radix et Rhizoma (Danshen) [31-33] ( $\odot$ Fig. 2).

For the assays, reference standards (Chemical Reference Standards CRS or Herbal Reference Standards HRS) have to be established by EDQM. For example, for the assay of Schisandrae chinensis fructus Ph.Eur. 6.3, Schisandrol A (= Wuweizichun A) has been established as a Chemical Reference Standard Schisandrin CRS.

Comparing the assay section in the ChP, it is obvious that in several monographs no minimum content for active or analytical markers is specified. In cases where appropriate methods are specified and minimum contents are provided, it is often a problem that reference compounds utilised for the assay of the ChP are not available at an acceptable price. In these cases, new analytical markers have to be selected, examined and validated by appropriate methods. In case pure compounds are not available, Herbal Reference Standards HRS will be used, like in Valerian root EP 6.8.

\section{Current Status of the EP Working Party on TCM Herbal Drugs \\ $\nabla$}

In the initial phase, the experts of the Phytochemistry Groups (13 A, 13 B) of the EP started work with the elaboration of TCM monographs. As a consequence of the heavy load of work for both Expert Groups, the EP Commission installed a TCM Working Party in 2008 with the only task to continue with the elaboration, evaluation and implementation of the urgently needed quality TCM herbal drug monographs. It was obvious that there was still a growing interest in the EU Member States to elaborate these quality monographs, since the import of TCM herbal drugs had increased considerably. Legally binding quality standards for TCM herbal drugs were very few at the time. General knowledge 
Table 3 Monographs of Chinese herbs accepted or published in the European Pharmacopoeia.

\begin{tabular}{ll} 
Bistortae rhizoma (Quan shen) & Ph. Eur. 6.0 \\
Notoginseng radix (San qi) & Ph. Eur. 6.0 \\
Sanguisorbae radix (Di yu) & Ph. Eur. 6.1 \\
Schisandrae fructus (Wu wei zi) & Ph. Eur. 6.3 \\
Carthami flos (Hong hua) & Ph. Eur. 6.4 \\
Ephedrae herba (Ma huang) & Ph. Eur. 6.7 \\
Stephaniae tetrandrae radix (Han Fangji) & Ph. Eur. 7.0 \\
\hline Astragali mongholici radix (Huang qi) & Ph. Eur. 7.0 \\
\hline Scutellariae baicalensis radix (Huang qin) & Ph. Eur. 7.1
\end{tabular}

about herbal drugs from TCM practise was still rather limited. Finally, many quality problems had been observed during the past, indicating a high risk potential for the patient if quality control is omitted. Consequently, inauguration of a specialised Working Party seemed to be necessary.

The original working programme of this TCM Working Party consisted of 83 monographs. Again, the elaboration should be carried out based on monographs of ChP 2005 and other relevant literature, such as the Japanese Pharmacopoeia and the Hong Kong Chinese Materia Medica Standards (HKCMMS), or the Chinese Drug Monographs and Analysis [11].

A precondition for all elaborations on TCM herbal drugs was the availability of commercial samples from the European market. As a minimum condition it was essential to have at least 5 to $8 \mathrm{com}$ mercial drug samples at our disposal, typical of material supplied to the market. These samples should be used for the confirmation of identification parameters and determining numerical values to be given in "Tests" and "Assays". Furthermore, authentic reference samples were needed to confirm that commercial samples are both genuine and of acceptable quality. In addition, reference samples of substitute/adulterant herbal drugs for exclusion tests should be available in sufficient quantity, to elaborate the respective methods for the "Test" section.

\section{Processed TCM Herbal Drugs}

Proc

The majority of herbal drugs used in traditional Chinese medicine appears to be subjected to some form of pretreatment (processing). In the context of the EP, these processed herbal drugs can be classified as "Herbal Drug Preparation". The current methods of processing have the potential to alter the appearance, the physical characteristics, and chemical constituents of a herbal drug considerably [30].

According to the ChP, "Processing is understood to make the crude drugs into small pieces through processing procedures such as cleaning, cutting, stir baking, so as to obtain the processed drugs fulfilling the requirements of therapy, dispensing and making preparations, thus assuring the safety and efficacy of the drugs". If one looks in the definition of the EP chapter "Herbal Drug Preparations", the methods typical for TCM herbal drugs are not listed so far, since the definition for "Preparations" for the EP includes only methods such as extraction, distillation, expression, fractionation, and purification. Consequently, "Herbal Drug Preparations" in the EP include extracts, essential oils, expressed juices, processed exudates and powdered herbal drugs for encapsulation.
Table 4 Monographs of Chinese herbs published in Pharmeuropa (Issue).

\begin{tabular}{ll}
\hline Arnebia radix (Zi cao) & 19.1 \\
\hline Citri reticulatae epicarpium et mesocarpium (Chen pi) & $19.1,22.4$ \\
\hline Eucommia ulmoidis bark (Du zhong) & 19.3 \\
\hline Sinomenii acuti caulis (Qing feng teng) & 19.3 \\
\hline Indigo naturalis (Qing dai) & 20.1 \\
\hline Polygoni multiflori radix (He shou wu) & $20.1,22.4$ \\
\hline Polygoni multiflori radix immutata (Shi he shou wu) & $20.1,22.4$ \\
\hline Puerariae lobatae radix (Ge gen) & 20.1 \\
\hline Puerariae thomsonii radix (Fen ge) & 20.1 \\
\hline Sophorae japonicae flos immaturus (Huai hua) & 21.2 \\
\hline Coicis semen (Yi yi ren) & 21.3 \\
\hline Acori calami rhizoma (Shui chang pu) & 21.1 \\
\hline Acori tatarinowii rhizoma (Shi chang pu) & 21.1 \\
\hline Acanthopanacis gracilistyli cortex (Wu jia pi) & 22.2 \\
\hline Angelicae dahuricae radix (Bai zhi) & 22.2 \\
\hline Angelicae pubescentis radix (Du huo) & 22.2 \\
Angelicae sinensis radix (Dang gui) & 22.2 \\
\hline Atractylodis macrocephalae rhizoma (Bai zhu) & 22.2 \\
\hline Atractylodis rhizoma (Cang zhu) & 22.2 \\
\hline Aucklandiae radix (Mu xiang) & 22.4 \\
\hline Drynariae rhizoma (Gu sui bu) & 22.4 \\
\hline Isatidis radix (Ban lan gen) & 22.2 \\
\hline Poria (Fu ling) & 22.3
\end{tabular}

Compared to these "Herbal Drug Preparations" of Western medicine, processed herbal drugs in TCM are quite different. Processing can be carried out in order to:

- obtain drugs of consistent size for better and constant effectiveness and better stability;

- reduce toxic or undersized effects;

- modify or increase the genuine effect of the herbal drug;

- modify the texture or molecular structure in order to increase solubility;

- prepare new herbal drugs by fermentation methods.

Herbal drugs can be treated by:

- mechanical processing;

- water for moistening the herbal drug prior to cutting;

- heat for altering the therapeutic properties;

- heat and water, or other liquids, like honey, saline, vinegar, wine or ginger juice, done by frying, boiling, or steaming with the liquids in question.

Against the background of these complex processing methods, a detailed chapter for these techniques may be established in order to be included in the EP as a "General Chapter". It will be important for the future implementation of TCM herbal drug monographs to be able to refer to the "Processing Methods" listed in this new "General Chapter". However, not all of the analytical requirements for the elaboration of herbal drug monographs may be appropriate for the respective processed herbal drug. This may necessitate the inclusion of additional tests, for example, the absence of rancidity when the plant material has been processed by stir baking in the presence of a vegetable oil. When herbal drugs such as aconite have been processed in order to reduce the content of toxic constituents, appropriate analytical methods to determine the acceptable limit values of the toxic compounds must be included [34-36]. 
Table 5 Pharmacopoeia or standards of various countries or regions that have monographic standards for CMM [40].

\begin{tabular}{|c|c|c|}
\hline Pharmacopoeia and monograph & Authority & Status \\
\hline WHO Monographs on Selected Medicinal Plants & WHO & unofficial \\
\hline Chinese Pharmacopoeia & SFDA China & official \\
\hline $\begin{array}{l}\text { Australian Regulatory Guidelines } \\
\text { for Complementary Medicines }\end{array}$ & TGA Australia & official \\
\hline European Pharmacopoeia & EDQM & official \\
\hline Hong Kong Chinese Materia Medica Standards & DHHK, China & official \\
\hline Japanese Pharmacopoeia & PA Japan & official \\
\hline Thai Herbal Pharmacopoeia & TFDA & official \\
\hline British Pharmacopoeia & BPC, UK & official \\
\hline American Herbal Pharmacopoeia (AHP) & private & unofficial \\
\hline
\end{tabular}

\section{Conclusion}

The development of Chinese medicine in Europe has reached a stage which requires precise studies and reliable quality control methods of the Materia Medica. Five years of intensive work of the Expert Groups resulted in a series of finalised and implemented monographs for the EP ( Table 3), furthermore, in a large number of published drafts ( $\odot$ Table 4 ), and many still pending working projects. Requirement is still the availability of sufficient samples, both commercial and authentic reference samples. Furthermore, the problems related to the pharmacological approach of appropriate assays to be included in the new concept of TCM herbal drug quality monographs should be resolved, since in most cases a defined pharmacological background requires precise studies of the Chinese Materia Medica and adaption to obtain reliable data on TCM drugs.

Because of the differences between ChP and EP, all new TCM herbal drugs for the EP had to be examined with the whole range of test methods available. In order to reduce contaminations, GACP rules have been established by WHO [37]. Even though a Good Agricultural Practice (GAP) system for the cultivation of Chinese herbs has been established, most Chinese herbs are not yet cultivated under controlled conditions, but are collected from their natural habitat or harvested from small cultivation bases [38]. Until June 2004 only 13 TCM herbs were grown in agricultural sites complying with GAP [39]. However, in the meantime the authorities have implemented GAP for 80 species [40].

Various countries or regions are currently establishing monographic standards for Chinese herbs (see O Table 5) [40]. Unfortunately, no harmonisation has been achieved so far. In order to reflect globalisation of TCM, a harmonised regulatory system would be desirable in order to improve quality and practicability.

\section{References}

1 Dobos GJ, Tan L, Cohen MH, McIntyre M, Bauer R, Li X, Bensoussan A. Are national quality standards for traditional Chinese herbal medicine sufficient? Current governmental regulations for traditional Chinese herbal medicine in certain Western countries and China as the Eastern origin country. Compl Ther Med 2005; 13: 183-190

2 Vlietinck A, Pieters L, Apers S. Legal requirements for the quality of herbal substances and herbal preparations for the manufacturing of herbal medicinal products in the European union. Planta Med 2009; 75: 683-688

3 Artiges A, Spieser JM. The role and future of the European Pharmacopoeia. Regul Affairs J 1994; (May-June): 349-351, 437-440

4 Artiges $A$. The role of pharmacopoeias in international harmonisation. J Pharm Biomed Anal 2001; 24: 769-772
5 European Directorate for the Quality of Medicines \& Health Care. European pharmacopoeia. Available at http://online.edqm.eu/entry.htm. Accessed November 2, 2010

6 Xiao P-G. Recent developments on medicinal plants in China. J Ethnopharmacol 1983; 7: 95-109

7 European Directorate for the Quality of Medicines \& Health Care. Technical Guide for The Elaboration of Monographs. Available at http:// www.edqm.eu/medias/fichiers/Technical_Guide_for_the_Elaboration _of_Monographs_on_herbal_drugs_and_herbal_drug_preparations_ 2007.pdf. Accessed November 2, 2010

8 Hsu HY. A Study of Chinese homonymic medicinal herbs. Oriental Healing Arts Int Bull 1987; 12: 387-408

9 Hu Y, Jiang Z, Leung KS, Zhao Z. Simultaneous determination of naphthoquinone derivatives in Boraginaceous herbs by high-performance liquid chromatography. Anal Chim Acta 2006; 577: 26-31

10 Zhao ZZ, Hu Y, Liang Z, Yuen JP, Jiang Z, Leung KS. Authentication is fundamental for standardization of Chinese medicines. Planta Med 2006; 72: 865-874

11 Wagner H, Bauer R, editors. Chinese drug monographs and analysis. Kötzting: Verlag für Ganzheitliche Medizin Dr. Erich Wühr $\mathrm{GmbH}$; 1996-2010

12 Chinese Pharmacopoeia Commission. TLC Atlas of Chinese Crude Drugs in Pharmacopoeia of the People's Republic of China. Beijing: People's Medical Publishing House; 2009

13 Zhong XK, Li DC, Jiang JG. Identification and quality control of Chinese medicine based on the fingerprint techniques. Curr Med Chem 2009; 16: 3064-3075

14 Yip PY, Chau CF, Mak CY, Kwan HS. DNA methods for identification of Chinese medicinal materials. Chin Med 2007; 2: 9

15 Carles M, Cheung MK, Moganti S, Dong TT, Tsim KW, Ip NY, Sucher NJ. A DNA microarray for the authentication of toxic traditional Chinese medicinal plants. Planta Med 2005; 71: 580-584

16 Techen N, Crockett SL, Khan IA, Scheffler BE. Authentication of medicinal plants using molecular biology techniques to compliment conventional methods. Curr Med Chem 2004; 11: 1391-1401

17 Kabelitz L. Zur mikrobiologischen Qualität von Arznei- und Gewürzdrogen. Sind die bisherigen Anforderungen sachgerecht? Z Arznei Gewürzpfl 2001; 6: 174-175

18 Kneifel W, Czech E, Kopp B. Microbial contamination of medicinal plants - a review. Planta Med 2002; 68: 5-15

19 Kabelitz L, Sievers $H$. Contaminants of medicinal and food herbs with a view to EU regulations. Innovations in Food Technol 2004; 1: 25-27

20 Leung KSY, Chan K, Chan CL, Lu GH. Systematic evaluation of organochlorine pesticide residues in Chinese materia medica. Phytother Res 2005; 19: 514-518

21 Kabelitz L. Heavy metals in herbal drugs. Eur J Herbal Med (Phytotherapy) $1998 ; 4: 1-9$

22 Chizzola R, Michitsch H, Franz C. Monitoring of metallic micronutrients and heavy metals in herbs, spices and medicinal plants from Austria. Eur Food Res Technol 2003; 216: 407-411

23 Gasser U, Klier B, Kühn AV, Steinhoff B. Current findings on the heavy metal content in herbal drugs. Pharmeuropa Sci Notes 2009; 1: 37-49

24 Nortier JL, Vanherweghem JL. Renal interstitial fibrosis and urothelial carcinoma associated with the use of a Chinese herb (Aristolochia fangchi). Toxicology 2002; 181-182: 577-580

25 Ioset JR, Raoelisona GE, Hostettmann K. Detection of aristolochic acid in Chinese phytomedicines and dietary supplements used as slimming regimens. Food Chem Toxicol 2003; 41: 29-36

26 Heinrich M, Chan J, Wanke S, Neinhuis C, Simmonds MS. Local uses of Aristolochia species and content of nephrotoxic aristolochic acid 1and 2-a global assessment based on bibliographic sources. J Ethnopharmacol 2009; 125: 108-144

27 Fu PP, Yang YC, Xia Q Chou MC, Cui YY, Lin G. Pyrrolizidine alkaloids tumorigenic components in Chinese herbal medicines and dietary supplements. J Food Drug Anal 2002; 10: 198-211

28 Roeder E. Medicinal plants in China containing pyrrolizidine alkaloids. Pharmazie 2000; 55: 711-726

29 Xue PS, Leung AY. Understanding the traditional aspect of Chinese medicine in order to achieve meaningful quality control of Chinese materia medica. J Chromatogr A 2008; 1216: 1913-1940

30 Liang YZ, Xie PS, Chan K. Chromatographic Fingerprinting and Metabolomics for Quality Control of TCM. Comb Chem High Throughput Screen, advance online publication 30 September 2010 
31 Liu AH, Lin YH, Yang M, Guo H, Guan SH, Sun JH, Guo DA. Development of the fingerprints for the quality of the roots of Salvia miltiorrhiza and its related preparations by HPLC-DAD and LC-MS(n). J Chromatogr B Analyt Technol Biomed Life Sci 2007; 846: 32-41

32 Cao J, Wei YJ, Qi LW, Li P, Qian ZM, Luo HW, Chen J, Zhao J. Determination of fifteen bioactive components in Radix et Rhizoma Salviae Miltiorrhizae by high-performance liquid chromatography with ultraviolet and mass spectrometric detection. Biomed Chromatogr 2008; 22: 164-172

33 Liu M, Li Y, Chou G, Cheng X, Zhang M, Wang Z. Extraction and ultra-performance liquid chromatography of hydrophilic and lipophilic bioactive components in a Chinese herb Radix Salviae Miltiorrhizae. J Chromatogr A 2007; 1157: 51-55

34 Csupor D, Wenzig EM, Zupkó I, Wölkart K, Hohmann J, Bauer R. Qualitative and quantitative analysis of aconitine-type and lipo-alkaloids of Aconitum carmichaeli roots. J Chromatogr A 2009; 1216: 2079-2086

35 Singhuber J, Zhu M, Prinz S, Kopp B. Aconitum in traditional Chinese medicine: a valuable drug or an unpredictable risk? J Ethnopharmacol 2009; 126: 18-30
36 Lu G, Dong Z, Wang Q Qian G, Huang W, Jiang Z, Leung KS, Zhao Z. Toxicity assessment of nine types of decoction pieces from the daughter root of Aconitum carmichaeli (Fuzi) based on the chemical analysis of their diester diterpenoid alkaloids. Planta Med 2010; 76: 825-830

37 World Health Organization. WHO guidelines on good agricultural and collection practices (GACP) for medicinal plants. Geneva: World Health Organization Geneva; 2003

38 Gao W, Duan H, Huang L, Xiao PG, Peak KY. Good agricultural practice (GAP) and sustainable resource utilization of Chinese materia medica. J Plant Biotech 2002; 4: 103-107

39 Siow YL, Gong Y, Au-Yeung KKW, Woo CWH, Choy PC. Emerging issues in traditional Chinese medicine. Can J Physiol Pharmacol 2005; 83: 321 334

40 Chan K, Leung KS, Zhao SS. Harmonization of monographic standards is needed to ensure the quality of Chinese medicinal materials. Chin Med 2009; $4: 18$ 